\section{Subproblem analysis of discrimination shift} learning in the turtle (Chrysemys picta picta)*

\author{
VIRGIL GRAF† and THOMAS TIGHE \\ Dartmouth College, Hanover, N.H. 03755
}

Turtles were given reversal and extradimensional shifts within a two-dimension (brightness and hue) two-pair discrimination task. Analysis of learning on the individual stimulus pairs during shifts indicated that the Ss learned the pairs as independent subproblems rather than as instances of a single problem.

A number of studies have found that infrahuman Ss learn an extradimensional shift (EDS), which requires response to a previously irrelevant dimension, more rapidly than they do a reversal shift (RS), which involves reversal of response to a previously relevant dimension. There is also a suggestion of a developmental trend in relative ease of the two types of shift, with young children finding reversal learning to be the more difficult problem (see Kendler \& Kendler, 1968, and Tighe \& Tighe, 1967 , for reviews of these data). Since these differences in shift performance suggest the operation of fundamentally different learning processes in different $S$ populations, there has been considerable interest in assessing the reliability of these comparative and developmental trends and in defining their significance for theories of learning and cognitive development.

Investigators in this area have focused almost exclusively on comparisons of overall trials or errors to criterion by groups undergoing the different types of shifts. However, Tighe, Glick, \& Cole (1971) have recently found that separate analyses of learning on the individual stimulus pairs (which they termed "subproblems") which comprise the shift tasks reveals patterns of behavior which appear to be basic to differences in shift learning. Essentially, application of the subproblem analysis to a conventional two-dimension two-pair RS-EDS comparison showed that 4-year-old children tended to learn the constituent stimulus pairs as independent subproblems, while 10-year-olds treated the pairs as instances of a single problem. The

*This research was supported by Grants EY-00355 and MH-11088 from the National Institutes of Health. Thomas Tighe is supported by Research Career Development Award K4-HD-43,859 from the National Institute of Child Health and Human Development.

torder of authorship was determined by flip of a coin. The assistance of Karl Frey and Scott $S$ pangenberg in collecting the data is gratefully acknowledged. former solution mode was found to be correlated with faster EDS than RS and the latter mode with fast RS.

In view of these observations on children's shift learning, it would be interesting to undertake similar analyses of RS-EDS comparisons in infrahuman Ss. However, studies of animal shift learning have not been conducted under procedures fully appropriate to application of the subproblem analysis, which requires that the same stimulus objects and pairs be presented throughout training. The present experiment meets these procedural requirements. Turtles were selected for study in order to add a new species to the comparative literature on shift learning while concurrently allowing extension of the subproblem analysis.

\section{METHOD}

The apparatus consisted of a black Plexiglas Y-maze. The stem and choice arms were $12.5 \mathrm{~cm}$ wide and the side and end walls, $15 \mathrm{~cm}$ high. The startbox, stem, and choice arms were 21,37 , and $18 \mathrm{~cm}$ long, respectively. The floors of the choice arms were $5 \mathrm{~cm}$ lower than the floor of the stem and were joined to the stem by $11-\mathrm{cm}$ 28-deg ramps. Translucent Plexiglas panels formed the rear walls of the endboxes. A $5-\mathrm{cm}$-sq light patch was projected from the rear onto the center of each panel. The light patches were generated from photic stimulators projected through interference wedges and were variable in two values of hue and two values of brightness. The wavelengths were $625 \mathrm{~nm}$ (orange) and $685 \mathrm{~nm}$ (red), the bandwidth for the interference wedges under these conditions being approximately $15 \mathrm{~nm}$. The amount of light available in the stimulator at each of the wavelengths was measured with a calibrated silicon photocell and multiplied by the spectral sensitivity of the turtle at these wavelengths (Graf, 1967), the product defining the luminance of the stimuli. The red stimulus was the least effective, and therefore the $685-\mathrm{nm}$ light with no auxiliary filters defined the red-bright condition. The orange-bright condition was defined by reducing the $625-\mathrm{nm}$ light in intensity with neutral density filters so that it was equal in luminance to the $685-\mathrm{nm}$ light. The dim condition for red and orange was obtained by introducing an additional $1.50 \mathrm{log}$ unit neutral density filter.

The $S$ was placed in the startbox for $15 \mathrm{sec}$, at which point the opaque startbox door was lifted and $S$ crawled to the choice point. Execution of choice was defined as descent of one of the 28-deg ramps. Entry into the endbox containing the $S+$ on that trial was reinforced by a bit of hamburger delivered on the rim of a magazine wheel which revolved in a corner of the endbox by means of a rotary solenoid. Concurrently, a 6.3-V magazine light located $10 \mathrm{~cm}$ above the magazine wheel was turned on for 10 sec. Following an incorrect choice, $S$ was left for $10 \mathrm{sec}$ in a darkened endbox. The intertrial interval was approximately $30 \mathrm{sec}$.

The stimulus pairs (red-bright vs orange-dim and red-dim vs orange-bright) were presented according to a Fellows sequence with regard to arm position of the pair members and order of pair presentation (Fellows, 1967). Ss received 20 trials per day under a noncorrection procedure and were run to a criterion of 18 correct responses on 2 successive days in both the original learning and shift phases. Half of the Ss underwent an RS and half an EDS. For example, an $\mathrm{S}$ who had orange as $\mathrm{S}+$ in the initial discrimination could be given either an RS to red as St or an EDS to bright or to $\operatorname{dim}$ as the $\mathrm{S}+$. Hue and brightness were used as the relevant dimension equally often within each shift condition. The irrelevant dimension was variable within trials throughout training.

The Ss were 16 Eastern painted turtles (Chrysemys picta picta) obtained from a dealer in southern New Jersey. A pilot study had indicated that shift training would be a difficult and lengthy process with these Ss. Accordingly, 16 Ss were selected from a total of 30 on the basis of performance during pretraining designed to shape eating and approach behavior in the apparatus. The major criteria of rejection were failure to eat or to make approach responses over the 4-day treatment. Ss selected then received 2 days of additional pretraining involving 20 reinforced approach responses per day.

RESULTS AND DISCUSSION

Factorial analysis of variance (Dimension Assignment by Shift Assignment) of trials to criterion in the initial discrimination did not yield significant $F$ values.

In regard to shift learning, the mean 


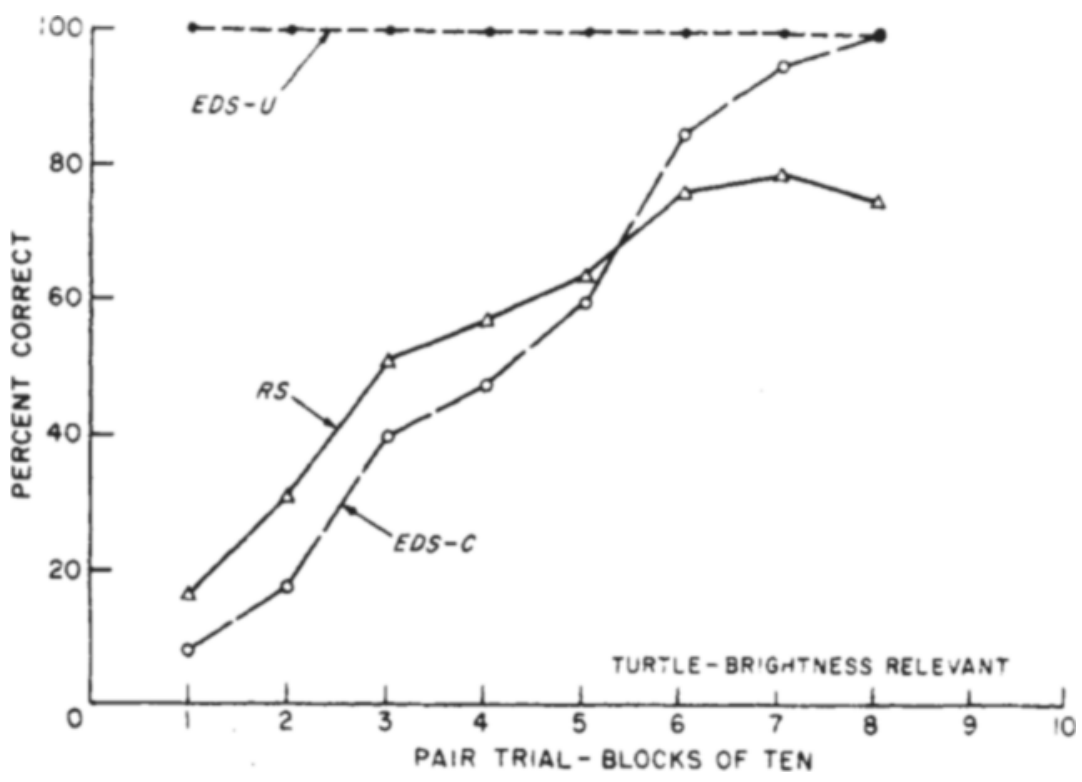

Fig. 1. Learning functions of turtles on EDS-C, EDS-U, and RS with brightness relevant. For RS, pair trial refers to successive presentations of both stimulus pairs.

number of trials to criterion in RS and in EDS with brightness relevant were 205 and 95 , respectively; the mean trials in RS and EDS with hue relevant were 315 and 320 , respectively. Factorial analysis of this measure revealed that EDS was learned more rapidly than $\mathrm{RS}(\mathrm{F}=8.18, \mathrm{df}=1 / 12$, $\mathrm{p}<.025$ ) and that learning was more rapid with brightness relevant than with hue relevant $(F=83.2, \mathrm{df}=1 / 12$, $\mathrm{p}<.001)$. A significant interaction between these factors was also obtained $(\mathrm{F}=9.81, \quad \mathrm{~d} f=1 / 12$, $p<.01)$. Analysis of the interaction by $t$ tests showed that EDS learning with brightness relevant proceeded faster than EDS learning with hue relevant $(t=3.31, \quad d f=6, \quad p<.02)$. None of the other pair comparisons approached significance.

The data of major concern in the experiment are shown in Figs. 1 and 2, which depict shift learning in terms of the subproblem analysis. In interpreting these figures, it should be recalled that an EDS involves reversal of the stimulus-reward relations on one of the stimulus pairs, the other pair retaining the reward relations which obtained in the initial discrimination. For RS, of course, stimulus-reward relations are reversed for both of the pairs. The figures show the trial-by-trial learning functions for the changed pair in extradimensional shift (EDS-C) and the unchanged pair (EDS-U), along with the averaged functions for the two pairs of reversal shift.

Considering, first, EDS with brightness relevant (Fig. 1), the EDS-C and EDS-U functions clearly indicate reversal learning. Viewed in this the faster EDS learning than RS pairs. learning exhibited on this dimension is seen as the outcome of the fact that RS involved two reversal subproblems, while EDS posed only one, each of which was learned at about the same rate.

In regard to EDS learning with hue as the relevant dimension, the degree of independent subproblem learning is not quite so dramatic as with brightness relevant. Nevertheless, independence is manifest in that performance on the unchanged pair is at or very near criterion ( $90 \%$ correct) throughout EDS learning, while there is a slow, gradual acquisition of correct response to the changed pair. Composite performance on the EDS subproblems is roughly equivalent to that on the RS composite subproblems, an outcome which the statistical analysis suggests reflects the extreme difficulty these Ss had in learning the changed EDS subproblem on the hue dimension. Therefore, it appears that the brightness relevant condition is the major contributor to the statistical main effect of faster EDS than RS in this experiment.

The pattern of subproblem learning observed in this experiment questions the conventional conception of "RS vs EDS learning" as a comparison of the difficulty of solving two distinct types of discrimination problems. For the species and task at hand, at least, RS vs EDS learning may be more accurately viewed as a comparison of the difficulty of reversing choice on the specific individual stimulus pairs or instances within each task.

The present data should be contrasted with Tighe, Glick, \& Cole's subproblem analysis (1971) of

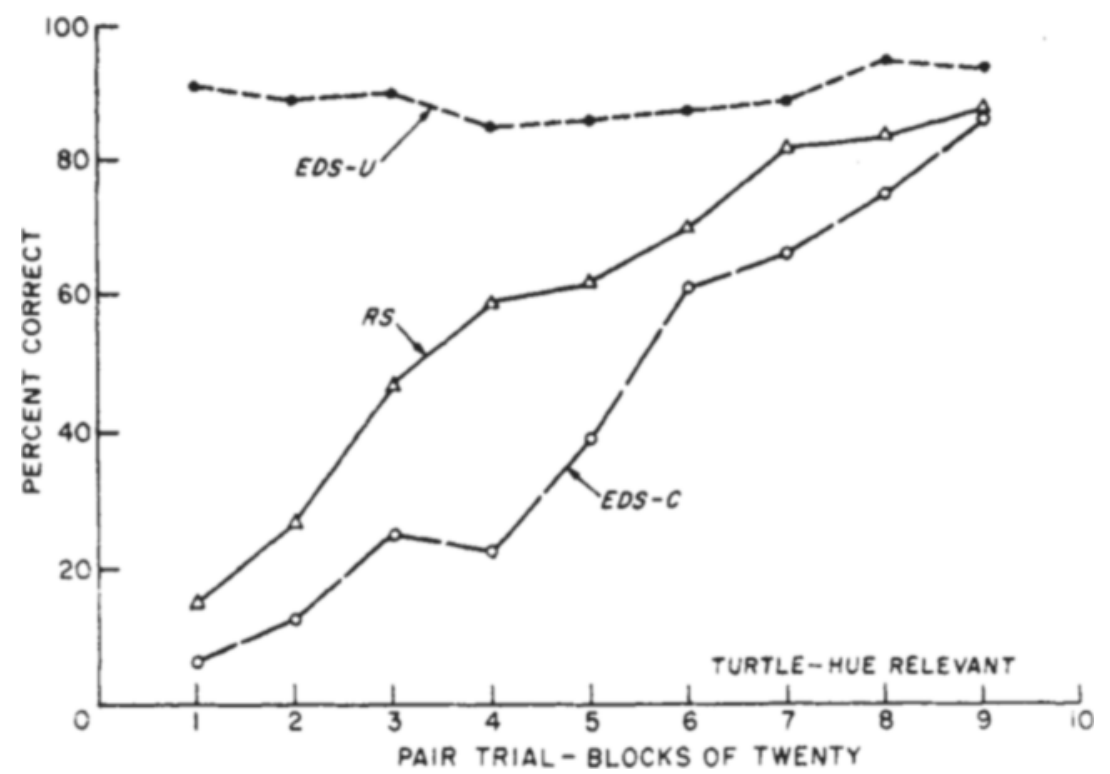

Fig. 2. Learning functions of turtles on EDS-C, EDS-U, and RS with hue relevant. For RS, pair trial refers to successive presentations of both stimulus 
RS-EDS learning by 4 - and 10-year-old children in a size and brightness task. In general respects, the RS, EDS-C, and EDS-U functions of the 4-year-olds resembled those of the turtles, although the EDS-U curve of the children was somewhat depressed relative to those observed here. In contrast, the 10-year-olds showed no evidence of independent subproblem learning. Their performance suffered markedly and equally on both EDS-C and EDS-U, and the learning functions for these pairs overlapped throughout shift. The composite RS curve showed the necessary initial drop but quickly surpassed the two EDS curves. Moreover, the dependency in learning the stimulus pairs was manifest from the onset of shift. Sixty-three percent of these Ss reversed their choice on the first postshift exposure to EDS-U after experiencing nonreward on EDS-C. That is, nonreward on EDS-C caused these Ss to change response to EDS-U, even though they had never experienced nonreward on this pair. Such "spontaneous reversal" of choice was virtually absent in the 4-year-olds and never appeared in turtles.

Subproblem analysis, then, revealed that 10 -year-olds learned the stimulus pairs as instances of a single problem, and for these Ss, RS was faster than EDS. On the other hand, the younger children and the turtles appeared to learn the pairs as independent subproblems, and for these Ss, RS was relatively difficult. Although further tests of the strength and generality of this correlation are clearly desirable, the present pattern of results bears noteworthy implications for theories of shift learning. They fit nicely the Kendler \& Kendler (1962) hypothesis that single-stage associations characterize the learning of infrahuman $S s$ and young children while dimensional mediators direct learning in the mature $S$. The results are also consistent with Tighe \& Tighe's (1966, 1971) assumption of a developmental trend from the learning of object-reward relations to the learning of dimension-reward relations in tasks of this nature. Finally, these data run counter to the assumption of attention theory (Sutherland, 1959), that discrimination learning is invariably based on selective response to dimensional features common to the task instances.

\section{REFERENCES}

FELLOWS, B. J. Chance stimulus sequences for discrimination tasks. Psychological Bulletin, 1967, 67, 87-92.

GRAF, V. A. A spectral sensitivity curve and wavelength discrimination for the turtle, Chrysemys picta picto. Vision Research, 1967, 7, 915-928.

KENDLER, H. H., \& KENDLER, T. S. Vertical and horizontal processes in problem-solving. Psychological Review, $1962,69,1-16$.

KENDLER, H. H., \& KENDLER, T. S. Mediation and conceptual behavior. In $\mathrm{K}$. W. Spence and J. T. Spence (Eds.), The psychology of learning and motivation. Vol, 2. New York: Academic Press, 1968. Pp. 197-244.

SUTHERLAND, N. S. Stimulus analysing mechanisms. In Proceedings of $a$ symposium on the mechanisation of thought processes. Vol. 2. London: Her Majesty's Stationery Office, 1959.

TIGHE, L. S., \& TIGHE, T. J. Discrimination learning: Two views in historical perspective. Psychological Bulletin, 1966, 66, 353-370.

TIGHE, T, J., GLICK, J., \& COLE, M Sub problem an alysis of discrimination-shift learning. Psychonomic Science, 1971, 24, 159-160.

TIGHE, T. J. \& \&IGHE, L. S. Discrimination shift performance of children as a function of age and shift procedure. Joumal of Experimental Psychology, 1967, 74, 466-470.

TIGHE, T. J., \& TIGHE, L. S. Stimulus control in children's learning. In A. D. Pick (Ed.), Minnesota symposia on child psychology. Vol. 6. Minnea polis: University of Minnesota, 1971, in press. 